Grav. Cosmol. No. 1, 2011

\title{
Exact solution of the relativistic magnetohydrodynamic equations in the background of a plane gravitational wave with combined polarization ${ }^{1}$
}

\author{
A. A. Agathonov and Yu. G. Ignatyev \\ Kazan State Pedagogical University, Mezhlauk str. 1, Kazan 420021, Russia
}

\begin{abstract}
We obtain an exact solution of the self-consistent relativistic magnetohydrodynamic equations for an anisotropic magnetoactive plasma in the background of a plane gravitational wave metric (PGW) with an arbitrary polarization. It is shown that, in the linear approximation in the gravitational wave amplitude, only the $\mathbf{e}_{+}$polarization of the PGW interacts with a magnetoactive plasma.
\end{abstract}

\section{Introduction}

In a series of previous articles by one of the authors (see, e.g., [1-3] a theory of gravimagnetic shock waves in a homogeneous magnetoactive plasma has been developed. The essence of this phenomenon is that a magnetized plasma in anomalously strong magnetic fields drifts under the action of gravitational waves $(\mathrm{GWs})$ in the $\mathrm{GW}$ propagation direction under the condition that the wave amplitude is large enough, and, on a certain wave front, the plasma velocity tends to the speed of light. Its energy density and the intensity of the frozen-in magnetic field then tend to infinity. In the subsequent papers this effect was proved on the basis of the kinetic theory, and the possibility of using this mechanism as an effective tool for detecting GWs from astrophysical sources was also shown. However, in all cited papers, a monopolarized gravitational wave was considered. In the present paper we consider the action of a GW with combined polarization on a magnetoactive plasma.

\section{Self-consistent RMHD equations in a gravitational field}

In [1], under the assumption that the dynamic velocity of the plasma $\left(v^{i}\right)$ is equal to that of the electromagnetic field ${ }^{2}$

$$
\stackrel{p}{T}_{i j} v^{j}=\varepsilon_{p} v_{i} ; \quad \stackrel{f}{T}_{i j} v^{j}=\varepsilon_{f} v_{i}, \quad(v, v)=1,
$$

a full self-consistent set of relativistic magnetohydrodynamic equations for a magnetized plasma in arbitrary gravitational field has been obtained. It consists of the

\footnotetext{
${ }^{1}$ Talk given at the International Conference RUDN-10, June 28 - July 3, 2010, PFUR, Moscow

${ }^{2}$ The index " $p$ " refers to the plasma, the index " $f$ " to the field, the comma denote a covariant derivative. The dynamic velocity of any kind of matter is, by definition, a timelike unit eigenvector of the energy-momentum tensor of this matter [4].
}

Maxwell equations of the first group

$$
\stackrel{*}{F}_{, k}^{i k}=0
$$

with the necessary and sufficient condition

$$
\begin{aligned}
\operatorname{Inv}_{1} & =F_{i j} F^{i j}=2 H^{2}>0, \\
\operatorname{Inv}_{2} & =F_{i j} F^{i j}=0,
\end{aligned}
$$

the Maxwell equations of the second group ${ }^{3}$ :

$$
F_{, k}^{i k}=-4 \pi J_{\mathrm{dr}}^{i}
$$

with a spacelike drift current

$$
J_{\mathrm{dr}}^{i}=-\frac{2 F^{i k} \stackrel{p}{T}_{k, l}^{l}}{F_{j m} F^{j m}}, \quad\left(J_{\mathrm{dr}}, J_{\mathrm{dr}}\right)<0
$$

and a conservation law for the total energy-momentum of the system

$$
T_{, k}^{i k}=\stackrel{p}{T}{ }_{, k}^{i k}+\stackrel{f}{T}_{, k}^{i k}=0 .
$$

The energy-momentum tensor (EMT) of the electromagnetic field, in the case of a coincidence of the plasma's and the field's dynamic velocities (1), is expressed through a pair of vectors, $v$ and $H$ [1]:

$$
\left.\stackrel{f}{T}{ }_{k}^{i}=-\frac{1}{8 \pi}\left[\left(\delta_{k}^{i}-2 v^{i} v_{k}\right) H^{2}+2 H^{i} H_{k}\right)\right] .
$$

The EMT of a relativistic anisotropic magnetoactive plasma in gravitational and magnetic fields is (see, e.g., [3])

$$
\stackrel{p}{T}^{i j}=\left(\varepsilon+p_{\perp}\right) v^{i} v^{j}-p_{\perp} g^{i j}+\left(p_{\|}-p_{\perp}\right) h^{i} h^{j},
$$

where $h^{i}=H^{i} / H$ is the spacelike unit vector of the magnetic field $((h, h)=-1) ; p_{\perp}$ and $p_{\|}$are the plasma pressures in the directions orthogonal and parallel to the magnetic field, respectively. 


\section{Solving the RMHD equations in the PGW metric}

Consider a solution of the Cauchy problem of the selfconsistent RMHD equations in the background of a vacuum gravitational-wave metric (see, e.g., $[5])^{4}$ :

$$
\begin{aligned}
d s^{2}= & 2 d u d v-L^{2}\left[\operatorname { c o s h } 2 \gamma \left(e^{2 \beta}\left(d x^{2}\right)^{2}\right.\right. \\
& \left.\left.+2 e^{-2 \beta}\left(d x^{3}\right)^{2}\right)-\sinh 2 \gamma d x^{2} d x^{3}\right]
\end{aligned}
$$

with homogeneous initial conditions on the null hypersurface $u=0$ :

$$
\beta(u \leq 0)=0 ; \quad \beta^{\prime}(u \leq 0)=0 ; \quad L(u \leq 0)=1,
$$

We assume the following:

- the plasma is homogeneous and at rest:

$$
\begin{aligned}
& v^{v}(u \leq 0)=v^{u}(u \leq 0)=1 / \sqrt{2} ; \\
& v^{2}=v^{3}=0 ; \quad \varepsilon(u \leq 0)=\stackrel{0}{\varepsilon} ; \\
& p_{\|}(u \leq 0)=\stackrel{0}{p}_{\|} ; \quad p_{\perp}(u \leq 0)=\stackrel{0}{\perp}_{\perp} ;
\end{aligned}
$$

- a homogeneous magnetic field is directed in the $\left(x^{1}, x^{2}\right)$ plane:

$$
\begin{aligned}
& H_{1}(u \leq 0)=\stackrel{0}{H} \cos \Omega \\
& H_{2}(u \leq 0)=\stackrel{H}{H} \sin \Omega \\
& H_{3}(u \leq 0)=0, \quad E_{i}(u \leq 0)=0
\end{aligned}
$$

where $\Omega$ is the angle between the axis $0 x^{1}$ (the PGW propagation direction) and the magnetic field $\mathbf{H}$.

The metric (10) admits the group of isometries $G_{5}$, associated with three linearly independent (at a point) Killing vectors

$$
\underset{(1)}{\xi^{i}}=\delta_{v}^{i} ; \quad \xi_{(2)}^{i}=\delta_{2}^{i} ; \quad \xi_{(3)}^{i}=\delta_{3}^{i} .
$$

Due to their existence in the metric (10), all geometric objects, including the Christoffel symbols, the Riemann tensor, the Ricci tensor and consequently the EMT of a magnetoactive plasma, are automatically conserved at motions along the Killing directions:

$$
\underset{\xi_{\alpha}}{\mathrm{L}} g_{i j}=0 \Rightarrow \underset{\xi_{\alpha}}{\mathrm{L}} R_{i j}=0 \Rightarrow \underset{\xi_{\alpha}}{\mathrm{L}} T_{i j}=0,
$$

${ }^{4} \beta(u)$ and $\gamma(u)$ are the amplitudes of the polarizations $\mathbf{e}_{+}$ and $\mathbf{e}_{\times}$, respectively; $u=\left(t-x^{1}\right) / \sqrt{2}$ is the retarded time, $v=\left(t+x^{1}\right) / \sqrt{2}$ is the advanced time. The PGW amplitudes are arbitrary functions of the retarded time $u$, and $L(u)$ is a background factor of the PGW. where $\underset{\xi}{\mathrm{L}} T_{i j}$ is a Lie derivative in the direction of $\xi$. We further require that the EMTs of the plasma $\stackrel{p}{T} i_{i j}$ and the electromagnetic field $\stackrel{f}{T}_{i j}$ inherit the symmetry separately. Thus all observed physical quantities $\mathbf{P}$ inherit the symmetry of the metric (10):

$$
\underset{\xi_{\alpha}}{\mathrm{L}} \mathbf{P}=0 \quad(\alpha=\overline{1,3}),
$$

i.e., taking into account the explicit form of the Killing vectors (14),

$$
\begin{aligned}
& p=p(u), \quad \varepsilon=\varepsilon(u), \quad v^{i}=v^{i}(u) \\
& F_{i k}=F_{i k}(u), \quad H_{i}=H_{i}(u), \quad h_{i}=h_{i}(u) .
\end{aligned}
$$

The vector potential agreeing with the initial conditions (13) is

$$
\begin{aligned}
& A_{v}=A_{u}=A_{2}=0 ; \\
& A_{3}=\stackrel{0}{H}\left(x^{1} \sin \Omega-x^{2} \cos \Omega\right) ; \quad(u \leq 0) .
\end{aligned}
$$

In the presence of a PGW, the vector potential becomes

$$
\begin{aligned}
& A_{2}=A_{v}=A_{u}=0 \\
& A_{3}=\stackrel{0}{H}\left(\frac{1}{\sqrt{2}}(v-\psi(u)) \sin \Omega-x^{2} \cos \Omega\right),
\end{aligned}
$$

where $\psi(u)$ is an arbitrary function of the retarded time, satisfying the initial condition

$$
\psi(u \leq 0)=u \text {. }
$$

Thus the magnetic field freezing-in condition in the plasma reduces to the two equalities

$$
\begin{aligned}
& v^{3}=0, \\
& \frac{1}{\sqrt{2}}\left(v_{v} \psi^{\prime}-v_{u}\right) \sin \Omega+v^{2} \cos \Omega=0 .
\end{aligned}
$$

The covariant components of the vector of magnetic field intensity relative to the Maxwell tensor are

$$
\begin{aligned}
& H_{v}=-\frac{0}{L^{2}}\left(v_{v} \cos \Omega+\frac{1}{\sqrt{2}} v^{2} \sin \Omega\right) \\
& H_{u}=\frac{0}{L^{2}}\left(v_{u} \cos \Omega-\frac{1}{\sqrt{2}} v^{2} \psi^{\prime} \sin \Omega\right), \\
& H_{2}=-\frac{1}{\sqrt{2}} \stackrel{0}{H} \cosh 2 \gamma e^{2 \beta} \sin \Omega\left(v_{v} \psi^{\prime}+v_{u}\right), \\
& H_{3}=\frac{1}{\sqrt{2}} \stackrel{0}{H} \sinh 2 \gamma \sin \Omega\left(v_{v} \psi^{\prime}+v_{u}\right) .
\end{aligned}
$$

The magnetic field intensity squared is

$$
H^{2}=\frac{H^{2}}{L^{4}}\left(L^{2} \psi^{\prime} \cosh 2 \gamma e^{2 \beta} \sin ^{2} \Omega+\cos ^{2} \Omega\right) .
$$


Using (23)-(27), the normalization relation for the velocity vector can be written in the equivalent form

$$
\begin{aligned}
& {\left[v_{v} \cos \Omega+v_{2} \frac{1}{\sqrt{2}} \sin \Omega\right]^{2}} \\
& =\frac{H^{2}}{H^{2}} v_{v}^{2} L^{4}-\frac{\sin ^{2} \Omega}{2} L^{2} \cosh 2 \gamma e^{2 \beta} .
\end{aligned}
$$

The components of the drift current are

$$
J_{\mathrm{dr}}^{i}=-\frac{1}{4 \pi L^{2}} \partial_{u}\left(L^{2} F^{i u}\right)
$$

Then,

$$
\begin{aligned}
J_{\mathrm{dr}}^{v} & =J_{\mathrm{dr}}^{u}=0, \\
J_{\mathrm{dr}}^{2} & =-\frac{H^{H} \sin \Omega}{2 \sqrt{2} \pi L^{2}} \cosh 2 \gamma \cdot \gamma^{\prime}, \\
J_{\mathrm{dr}}^{3} & =-\frac{{ }_{H}^{H} \sin \Omega e^{2 \beta}}{2 \sqrt{2} \pi L^{2}}\left(\sinh 2 \gamma \cdot \gamma^{\prime}+\cosh 2 \gamma \cdot \beta^{\prime}\right) .
\end{aligned}
$$

Because of existence of the isometries (14), we obtain the following integrals [1]:

$$
L^{2} \underset{(\alpha)}{\xi^{i}} T_{v i}=C_{a}=\mathrm{const} \quad(\alpha=\overline{1,3}) .
$$

We consider only the case of transverse $P G W$ propagation $(\Omega=\pi / 2)$. Then, substituting the expressions for the plasma and electromagnetic field EMT into the integrals (33), using the relations (26)-(28) and also the initial conditions (11), we bring the integrals of motion to the form

$$
\begin{aligned}
& 2 L^{2}\left(\varepsilon+p_{\|}\right) v_{v}^{2}-\left(p_{\|}-p_{\perp}\right) \frac{\stackrel{H}{H}^{2}}{H^{2}} \cosh 2 \gamma e^{2 \beta} \\
& =(\stackrel{0}{\varepsilon}+\stackrel{0}{p}) \Delta(u), \\
& L^{2}\left(\varepsilon+p_{\|}\right) v_{v} v_{2}=0, \\
& L^{2}\left(\varepsilon+p_{\|}\right) v_{v} v_{3}=0,
\end{aligned}
$$

where

$$
\stackrel{0}{p}=\stackrel{0}{p}+
$$

and the so-called governing function of the GMSW is introduced:

$$
\Delta(u)=1-\alpha^{2}\left(\cosh 2 \gamma e^{2 \beta}-1\right),
$$

with the dimensionless parameter $\alpha^{2}$,

$$
\alpha^{2}=\frac{\stackrel{0}{H^{2}}}{4 \pi(\stackrel{0}{\varepsilon}+\stackrel{0}{p})} .
$$

Solving (34) with respect to $v_{v}$, we obtain expressions for the components of the velocity vector as functions of the scalars $\varepsilon, p_{\|}, p_{\perp}, \psi^{\prime}$ and explicit functions of the retarded time:

$$
\begin{aligned}
v_{v}^{2}= & \frac{\left({ }^{0} p\right)}{2 L^{2}\left(\varepsilon+p_{\|}\right)} \Delta(u) \\
& +\frac{\left(p_{\|}-p_{\perp}\right)}{\left(\varepsilon+p_{\|}\right)} \frac{H^{2}}{H^{2}} \frac{\cosh 2 \gamma e^{2 \beta}}{2 L^{2}} .
\end{aligned}
$$

From (35), (36) we get:

$$
v_{2}=v_{3}=0 .
$$

We obtain the component $v_{u}$ from the normalization relation for the velocity vector, using (40) and (41):

$$
v_{u}=\frac{1}{2 v_{v}},
$$

and from the freezing-in condition (22) we get the value of the derivative of potential $\psi^{\prime}$ :

$$
\psi^{\prime}=\frac{1}{2 v_{v}^{2}}
$$

Using it, the scalar $H^{2}$ is determined from the relation (27):

$$
H^{2}=\frac{\stackrel{0}{H}^{2}}{L^{2}} \frac{\cosh 2 \gamma e^{2 \beta}}{2 v_{v}^{2}} .
$$

From the RMHD set of equations it is possible to obtain the following differential equation in the PGW metric:

$$
\begin{aligned}
L^{2} \varepsilon^{\prime} v_{v} & +\left(\varepsilon+p_{\|}\right)\left(L^{2} v_{v}\right)^{\prime} \\
& +\frac{1}{2} L^{2}\left(p_{\|}-p_{\perp}\right) v_{v}\left(\ln H^{2}\right)^{\prime}=0 .
\end{aligned}
$$

To solve this equation, it is necessary to impose two additional relations between the functions $\varepsilon, p_{\|}$, and $p_{\perp}$, i.e., an equation of state:

$$
p_{\|}=f(\varepsilon) ; \quad p_{\perp}=g(\varepsilon) .
$$

\section{Barotropic equation of state}

Consider a barotropic equation of state of the anisotropic plasma, where the relations (46) are linear:

$$
p_{\|}=k_{\|} \varepsilon ; \quad p_{\perp}=k_{\perp} \varepsilon .
$$

Equation (45) is easily integrated under the conditions (47), and we get one more integral:

$$
\varepsilon\left(\sqrt{2} L^{2} v_{v}\right)^{\left(1+k_{\|}\right)} H^{\left(k_{\|}-k_{\perp}\right)}=\stackrel{0}{=} \stackrel{0}{H^{(}}{ }^{\left(k_{\|}-k_{\perp}\right)} .
$$

In the case of a barotropic equation of state under the conditions (47), substitution of (44) into (40) results in

$$
v_{v}^{2}=\frac{1}{2} \frac{\stackrel{0}{\varepsilon}^{2}}{L^{2} \varepsilon} \Delta(u) .
$$


Substituting (44) and (49) into (48), we obtain a closed equation with respect to the variable $\varepsilon$, whose solution gives:

$$
\begin{aligned}
& \varepsilon={ }^{\varepsilon}\left[\Delta^{1+k_{\perp}} L^{2\left(1+k_{\|}\right)}\left(\cosh 2 \gamma e^{2 \beta}\right)^{k_{\|}-k_{\perp}}\right]^{-g_{\perp}}, \\
& v_{v}=\frac{1}{\sqrt{2}}\left[\Delta L^{\left(k_{\|}+k_{\perp}\right)}\left(\cosh 2 \gamma e^{2 \beta}\right)^{\frac{k_{\|}-k_{\perp}}{2}}\right]^{g_{\perp}}, \\
& H=\stackrel{0}{H}\left[\Delta L^{\left(1+k_{\|}\right)}\left(\cosh 2 \gamma e^{2 \beta}\right)^{-\frac{1-k_{\|}}{2}}\right]^{-g_{\perp}},
\end{aligned}
$$

where

$$
g_{\perp}=\frac{1}{1-k_{\perp}} \in[1,2] .
$$

In particular, for an ultrarelativistic plasma with zero parallel pressure,

$$
k_{\|} \rightarrow 0 ; \quad k_{\perp} \rightarrow \frac{1}{2}
$$

we obtain from (50)-(53):

$$
\begin{aligned}
& v_{v}=\frac{1}{\sqrt{2}} L \Delta^{2}\left(\cosh 2 \gamma e^{2 \beta}\right)^{-1 / 2}, \\
& \varepsilon=\stackrel{0}{\varepsilon} L^{-4} \Delta^{-3}\left(\cosh 2 \gamma e^{2 \beta}\right), \\
& H=\stackrel{0}{H} L^{-2} \Delta^{-2}\left(\cosh 2 \gamma e^{2 \beta}\right) .
\end{aligned}
$$

\section{The energy balance equation}

In [1], it has been shown that the singular state, which exists in a magnetized plasma under the condition $2 \beta_{0} \alpha^{2}>1$ on the hypersurface

$$
\Delta\left(u_{*}\right)=0,
$$

is removed using the back reaction of the magnetoactive plasma on the GW. That leads to efficient absorption of GW energy by the plasma and a restriction on the GW amplitude. A qualitative analysis of this situation can be carried out using a simple model of energy balance proposed in [2]. The energy flow of the magnetoactive plasma is directed along the PGW propagation direction, i.e., along the $x^{1}$ axis. Let $\beta_{*}(u)$ and $\gamma_{*}(u)$ be the vacuum PGW amplitudes. In the WKB approximation,

$$
8 \pi \varepsilon \ll \omega^{2},
$$

where $\omega$ is the characteristic PGW frequency and $\varepsilon$ is the matter energy density, all functions still depend on the retarded time only (see [6]). Thus $\beta(u)$ and $\gamma(u)$ are the PGW amplitudes subject to absorption in plasmas. The local energy conservation law should be satisfied:

$$
T^{41}(\beta, \gamma)+\stackrel{g}{T}^{41}(\beta, \gamma)=\stackrel{g}{T} 41\left(\beta_{*}, \gamma_{*}\right),
$$

where $\stackrel{g}{T}^{41}(\beta, \gamma)$ is the energy flow of a weak GW in the direction $0 x^{1}$ (see [7]).

In the case of transversal PGW propagation and with a barotropic equation of state of an anisotropic plasma, using the solutions of magnetohydrodynamics and Eqs. (50), (51), (52) with the dimensionless parameter $\alpha^{2}$ (39), one can obtain the energy balance equation in the form

$$
\begin{aligned}
& \frac{\stackrel{0}{H}^{2}}{4}\left(\Delta^{-4 g_{\perp}}-1\right)\left(\frac{1}{\alpha^{2}}+1\right) \\
& \quad+\left(\gamma^{\prime}\right)^{2}+\left(\beta^{\prime}\right)^{2}=\left(\gamma_{*}^{\prime}\right)^{2}+\left(\beta_{*}^{\prime}\right)^{2} .
\end{aligned}
$$

Since, in a linear approximation by smallness of the amplitudes $\beta$ and $\gamma$, the governing function (38) does not depend on the function $\gamma(u)$,

$$
\Delta(u)=1-2 \alpha^{2} \beta+O\left(\beta^{2}, \gamma^{2}\right),
$$

and the functions $\beta(u), \gamma(u)$ are arbitrary and functionally independent, then, up to $\beta^{2}, \gamma^{2}$, the relation (61) can be split into two independent parts:

$$
\begin{aligned}
& 2 \stackrel{0}{H^{2}} g_{\perp}\left(1+\alpha^{2}\right) \beta+\left(\beta^{\prime}\right)^{2}=\left(\beta_{*}^{\prime}\right)^{2}, \\
& \left(\gamma^{\prime}\right)^{2}=\left(\gamma_{*}^{\prime}\right)^{2} .
\end{aligned}
$$

Here, according to the meaning of the local energy balance equation, we consider short gravitational waves (59), so we can neglect the squares of the PGW amplitudes as compared with the squares of their derivatives with respect to the retarded time. Thus, according to (64),

$$
\gamma_{*}(u)=\gamma(u),
$$

i.e., in the linear approximation, a weak gravitational wave with the polarization $\mathbf{e}_{\times}$does not interact with a magnetized plasma. This coincides with the conclusion of the paper [8]. Thus the energy balance equation takes the form obtained in [3]:

$$
\dot{\Delta}^{2}+\xi^{2} \Upsilon^{2}\left[\Delta^{-4 g_{\perp}}-1\right]=\Upsilon^{2} \sin ^{2}(s),
$$

where $\xi^{2}$ is the so-called first parameter of the GMSW [2]:

$$
\begin{aligned}
& \xi^{2}=\frac{\stackrel{H}{H}^{2}}{4 \beta_{0}^{2} \omega^{2}}, \\
& \Upsilon=2 \alpha^{2} \beta_{0}
\end{aligned}
$$

- the second GMSW parameter. The dot denotes differentiation with respect to the dimensionless time variable $s$

$$
s=\sqrt{2} \omega u .
$$




\section{Conclusion}

Thus we have obtained a generalization of the results of [1]- [3] to gravitational waves with two polarizations and showed that, in the linear approximation, the polarization $\mathbf{e}_{\times}$does not interact with a magnetized plasma. This justifies the applicability of the previously obtained results for arbitrarily polarized gravitational waves.

\section{References}

[1] Yu. G. Ignat'ev, Grav. Cosmol. 1, 287 (1995).

[2] Yu. G. Ignat'ev, Grav. Cosmol. 2, 345 (1996).

[3] Yu. G. Ignat'ev and D. N. Gorokhov, Grav. Cosmol. 3, 261 (1997).

[4] J. L. Synge, Relativity: The General Theory (NHPC, Amsterdam, 1960).

[5] C. W. Misner, K. S. Thorne, and J. A. Wheeler, Gravitation (Freeman, San Francisco, 1973).

[6] Yu. G. Ignat'ev and A. B. Balakin., Sov. Phys. J. (Izv. Vuzov, Fizika) 24, No. 7, 20 (1981).

[7] L. D. Landau and E. M. Lifshitz, Field Theory (Nauka, Moscow, 1973).

[8] Yu. G. Ignat'ev and N. R. Khusnutdinov, Ukr. Fiz. Zh. No. 31, 707 (1986). 